few shreds which were all that remained of the recti muscles, the external canthus having been divided. Very free haemorrhage followed, which was easily controlled by pressure and gauze packing.

On examination of the globe a deep furrow was seen in the sclera just behind the limbus, due to the pressure of the lids. On section a choroidal haemorrhage $\frac{1}{8}$ in. thick was found extending all round. The retina was everywhere in position, and there was no sign of a new growth.

As post-operative treatment, first dry, and later moist dressings were applied to the remaining mass with no effect. The previous soft swelling was gradually replaced by a harder one, and about a fortnight later I took a section of it and sent it to the pathologist who diagnosed it as " spindle-celled sarcoma."

On December 17 a typical exenteration of the orbit was performed, the lids being included. At a suspicious place on the orbital plate of the superior maxilla a portion of bone was removed. Finally, the skin was sutured to the periosteum, and the orbit packed with gauze for a few days, when the latter was replaced by a surface dressing. The orbit was allowed to fill up with granulations, whilst the epithelium grew backwards, and finally covered the surface of the former. The patient was discharged from hospital on February 18, 1919, the process of healing being nearly complete.

From a cosmetic point of view the result is very good.

When communicated with recently the patient was in good health, and there was as yet no sign of a local or general recurrence of the disease.

The extremely short history given by the patient is surprising, and no amount of questioning revealed a longer one. Examination of the exenterated mass gave no clue to the site of origin of the growth, which, I presume, arose from some of the orbital tissues.

\title{
PAPILLITIS DUE TO TONSILLITIS: THREE CASES BY
}

P. A. HARRY, M.D., OPHTHALMIC SURGEON, ROCHDALE INFIRMARY

PyorRhoea is not an uncommon cause of eye disease, and although this condition is often associated with tonsillitis it is unusual for the latter alone to produce optic neuritis.

The principal features are :-Intensity of the papillitis; completeness with which papillitis cleared up; the unilateral character. 
Mr. B-, aged 35, gave a history of sudden diminution of vision in right eye, severe frontal headache. Examination showed dilated pupil and intense optic neuritis in R.E., there was no P.L. L.V. $6 / 6$, left field normal.

Investigation of cause showed that patient was in perfect condition, formerly suffered from rheumatism, nasal sinuses apparently normal, teeth artificial, both tonsils presented appearance of chronic follicular tonsillitis. Immediate removal by enucleation advised. The headache was more intense after removal, with increase rather than abatement of neuritis. Administration of large doses of sodium salicylate produced diminution of the papillitis with complete cure and restoration of vision in two weeks.

Miss M., aged 30, gave a history of having suffered from many attacks of quinsy. Recently had partial removal of tonsils performed; now complains of loss of sight ; R.V. barely 6/60, L.V. normal, refraction hypermetropic. Right disc swollen, edges illdefined, whitish exudate noticed; left fundus normal. No cause discovered for the unilateral neuritis, except for post-operative tonsillitis. Treatment sod. salicyl.

Miss Agnes W., age 27, single, gave a history of severe headache, worse in left temporal region, duration three weeks, onset sudden, has been treated by family doctor with powders which only gave temporary relief. Ptosis left, slight at first becoming worse during last week, patient still at work (shop assistant). Ocular examination showed dilated pupil left, well marked papillitis, area of disc increased, R.V. 6/6, L.V. hand movements, slight tenderness on pushing left eye backwards in orbit. Urine normal.

No nasal disease, patient rheumatic but otherwise healthy, no pyrorrhoea, both tonsils enlarged and septic purulent material could be squeezed out of the follicles. Operation of tonsillectomy refused. Large doses of sodium salicylate prescribed. After ten days the headache disappeared and ptosis less marked. Margins of disc still obscured and irregular, swelling less marked, no exudate. L.V. 6/18. Patient discontinued treatment without permission and although a holiday was advised she would not take one. Six weeks after her first visit she returned with history of renewed headaches not markedly temporal, loss of vision right, R.V. hand movements, L.V. 6/6, no error of refraction. Intense optic neuritis right eye, congestion of left disc but no papillitis, moderate ptosis right. Exacerbation of follicular tonsillitis, tonsil plus 3 , and decidedly inflamed. Treatment with sodium salicylate commenced and left tonsil dried and carefully swabbed with 10 per cent. solution of silver nitrate. In three weeks all symptoms had disappeared, vision perfectly normal, no ptosis, fundi normal. 